\title{
Postoperative Management of Lower Extremity Free Tissue Transfer: A Systematic Review
}

\author{
Carol E. Soteropulos, MD ${ }^{1}$ Jenny T. Chen, MD $\quad$ Samuel O. Poore, MD, PhD ${ }^{1} \quad$ Catharine B. Garland, MD ${ }^{1}$ \\ ${ }^{1}$ Division of Plastic Surgery, Department of Surgery, University of \\ Wisconsin School of Medicine and Public Health, Madison, Wisconsin \\ J Reconstr Microsurg 2019;35:1-7.

\begin{abstract}
Address for correspondence Catharine B. Garland, MD, Division of Plastic and Reconstructive Surgery, Department of Surgery, University of Wisconsin School of Medicine and Public Health, 600 Highland Avenue, G5/361 CSC, Madison, WI 53792-3236 (e-mail: garland@surgery.wisc.edu).
\end{abstract}

\section{Abstract \\ Keywords \\ - free tissue transfer \\ - lower extremity reconstruction \\ - lower extremity free flap \\ - dangle protocol \\ - progressive dependency \\ - compressive wrapping}

Background Free tissue transfer for lower extremity reconstruction is a safe and reliable option for a wide range of challenging wounds; however, no consensus exists regarding postoperative management.

Methods A systematic review of postoperative management of lower extremity free tissue transfer was conducted using Medline, Cochrane Database, and Web of Science. Multicenter surveys, randomized controlled trials, cohort studies, and case series were reviewed.

Results Fifteen articles investigating current protocols, flap physiology, and aggressive dangle protocols were reviewed. The following evidence-based conclusions were made: (1) Free tissue transfer to the lower extremity is unique due to altered hemodynamics and dependency during orthostasis. Free flap circulation is dependent on locally mediated responses and deprived of compensatory muscular and neurovascular mechanisms that prevent venous congestion in the normal extremity. (2) Compressive wrapping reduces venous congestion and edema and may induce ischemic conditioning, which can increase blood flow. (3) Dangle protocols vary widely in timing of initiation, frequency, and monitoring. Small volume studies examining aggressive mobilization protocols initiating early dependency have led to earlier ambulation and discharge, with no change in flap survival as compared with conservative protocols. (4) Weight bearing may begin after the completion of dangle protocol if no orthopedic injury is present. Conclusions Early initiation of a dangle protocol does not appear to negatively impact flap survival based on this systematic review. Compressive wrapping may be a useful adjunct. Many surgeons agree that clinical monitoring is sufficient; there is no consensus on the utility of adjunct monitoring techniques. Weight bearing may begin after completion of dangle protocol with close flap monitoring, if not prevented by orthopedic restrictions. By providing additional outflow vasculature to reduce venous congestion, flow-through anastomoses may eliminate the need for a dangle protocol. Further research, including large randomized controlled trials is still needed to establish high-level evidence-based conclusions. received

January 19, 2019

accepted after revision

May 22, 2018

published online

July 29, 2018
Copyright $\odot 2019$ by Thieme Medical Publishers, Inc., 333 Seventh Avenue, New York, NY 10001, USA. Tel: +1(212) 584-4662.
DOI https://doi.org/ 10.1055/s-0038-1667049. ISSN 0743-684X. 
Free tissue transfer is a well-established, safe and reliable option for coverage of complex wounds in all parts of the body. In the lower extremity, free tissue transfer is commonly utilized for the reconstruction of posttraumatic wounds, and defects left after resection of bony and soft tissue malignancies. Despite the relatively high success rates of free tissue transfer, failure rates of more than $10 \%$ have been reported in the lower extremity in some series, especially traumatic reconstruction. ${ }^{1-3}$ In addition to the risks of venous and arterial thrombosis, lower extremity free flaps are located in a dependent position on the body, which increases complications. ${ }^{4}$ Due to limited venous outflow, the flap is at risk of venous congestion and tissue edema, which lower the perfusion pressure of the flap and increase the risk of failure. ${ }^{3,5}$ Employing a "dangle protocol" has been shown to balance these issues during the early postoperative period when the flap is deprived of collateral vasculature and robust musculature to aid with venous outflow.

Although the majority of reconstructive surgeons agree that a dangle protocol is necessary to ensure a successful outcome, considerable variability is encountered among such protocols, even within institutions. ${ }^{6}$ This variability indicates that multiple successful management strategies exist, and that there is opportunity for further research to develop evidence-based recommendations. The objectives of this review are to (1) describe the physiologic mechanisms contributing to a higher free flap failure rate in the lower extremity; (2) provide a comprehensive discussion of successful protocols currently in use at major institutions, and (3) discuss newer operative techniques and protocols that may shorten hospital stay and improve overall functional outcomes.

\section{Methods}

A systematic review of postoperative management of lower extremity free tissue transfer was conducted using the PRISMA guidelines. ${ }^{7}$ Medline, the Cochrane database, and Web of Science were searched from earliest available date through November 2017. Search terms included the following: lower extremity free tissue transfer, dangle protocol, dangle lower extremity, lower extremity flap postoperative management, and lower extremity flap training. Inclusion criteria comprised surveys, case series, cohort studies, and randomized controlled trials examining the postoperative management of free tissue transferred to the lower extremity of human patients. All tissue types and anastomoses types were included. Exclusion criteria included non-English language studies, cadaver studies, and animal trials. After reviewing abstracts, full articles were reviewed and selected based on their relevance to the above objectives. Articles without a complete description of postoperative management protocols including monitoring, dangling, compressive wrapping, and ambulation were excluded. Studies describing the physiology of dangling were also reviewed. Literature search and article selection was performed independently by two study authors and then compared for final article inclusion.

\section{Results}

1131 records were identified through database searching of the above-listed criteria after duplicates were removed. A total of 210 records were screened by title, and 63 screened further by abstract to include those relevant to this review. Of the 41 full-text articles that were assessed for eligibility, 26 of these were excluded because they did not meet the inclusion criteria stated earlier. After complete review, 15 articles were selected for inclusion ( $\mathbf{- F i g . 1}$ ). These included three multiinstitution surveys, two randomized controlled trials, nine cohort studies, and one case series.

Three surveys examined postoperative management protocols for free tissue transfer to the lower extremity from both the United States and United Kingdom. ${ }^{8-10}$ These surveys looked at initiation, timing, advancement, and conclusion of dangle protocols, as well as flap monitoring, compressive wrapping, and time to weight bearing (-Figs. 2 and 3). The surveys studied demonstrated significant variation of postoperative flap management including protocol variability on a case-by-case basis. Less than $10 \%$ of surgeons responded that they do not routinely use dangle protocols as a part of lower extremity free flap management. Compressive wrapping was addressed by Rohde et al. All five surgeons surveyed recommended wrapping. Initiation of wrapping varied as early as with initial dangle, to 4 to 6 weeks postoperatively when the flap was considered mature. ${ }^{6}$ Weight bearing was typically started at the conclusion of the dangle protocol, presuming there was no concomitant orthopedic injury that would prevent this. Time to weight bearing varied with protocol length, although non-weight-bearing crutch ambulation was safely started at the conclusion of most dangle protocols. In the instance of bony injury, recommendations for weight bearing were dictated by the orthopedic surgeon managing the fractures.

Twelve other studies examined the physiology and safety of free tissue transferred to the lower extremity with early initiation of dangle (-Table 1)..$^{1,3,10-19}$ These 12 studies comprised 10 author groups and 229 patients. Data were extracted from these studies in the following categories: study design, number of patients, flap type (donor site, tissue type, and anastomosis characteristics), flap failure rate, dangle initiation, dangle advancement, dangle end point, flap monitoring techniques, use of compressive wrapping, time to ambulation, and time to weight bearing.

Invasive and noninvasive technologies were used in addition to clinical evaluation to examine flap physiology following transfer to the lower extremity. These included implanted arterial and venous catheters, microdialysis evaluation of metabolic end products, spectroscopy, interstitial compartment pressure measurement, laser Doppler, and ultrasound.

Dangle was initiated between postoperative day two and postoperative day seven in all author groups utilizing traditional anastomotic techniques.,10-17,19 Miyamoto et al initiated dangle as early as postoperative day one with the use of flow-through anastomoses. ${ }^{18}$ Seth et al advocated for safe initiation of dangle on postoperative day two, which was 


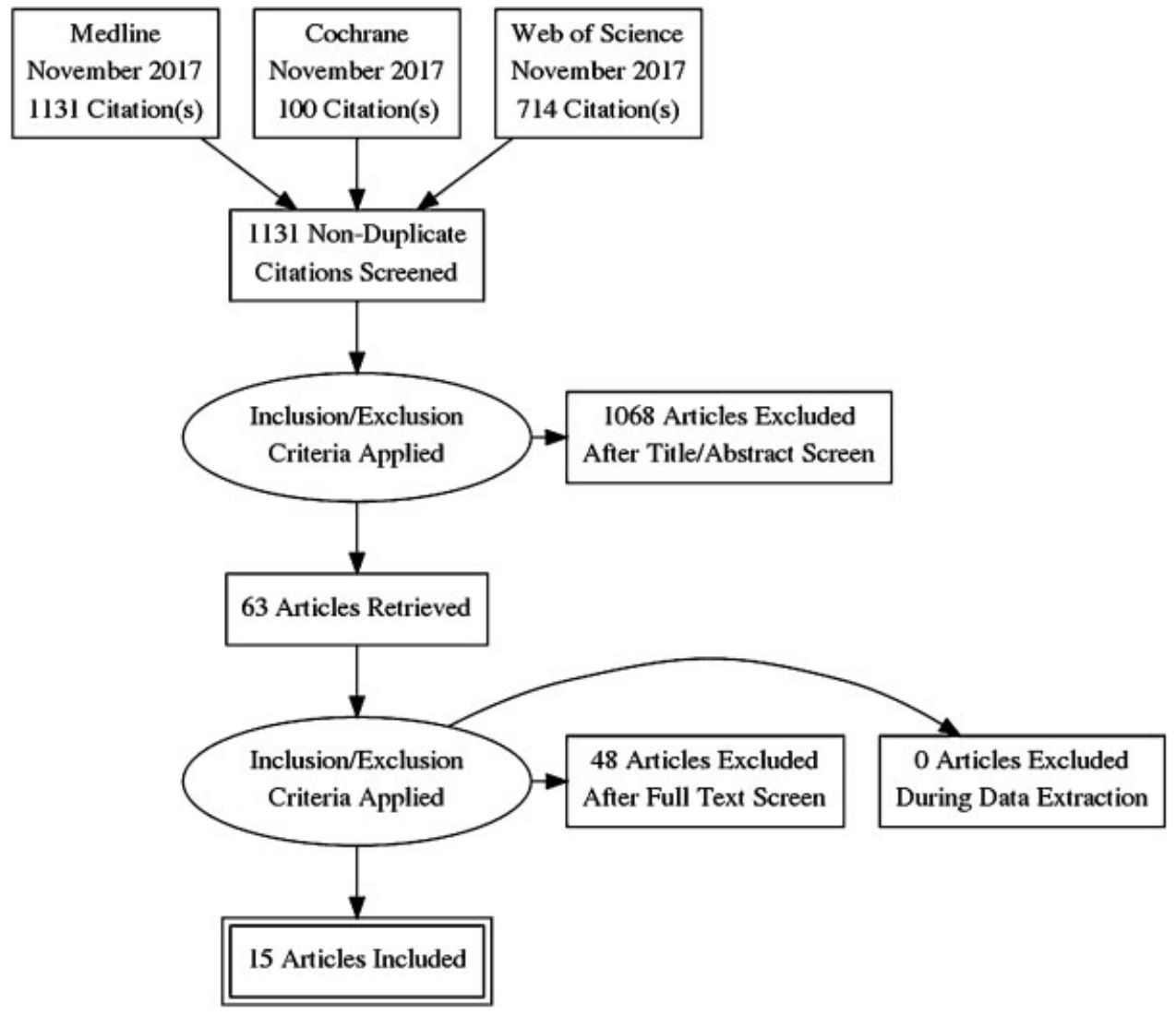

Fig. 1 PRISMA inclusion guidelines.

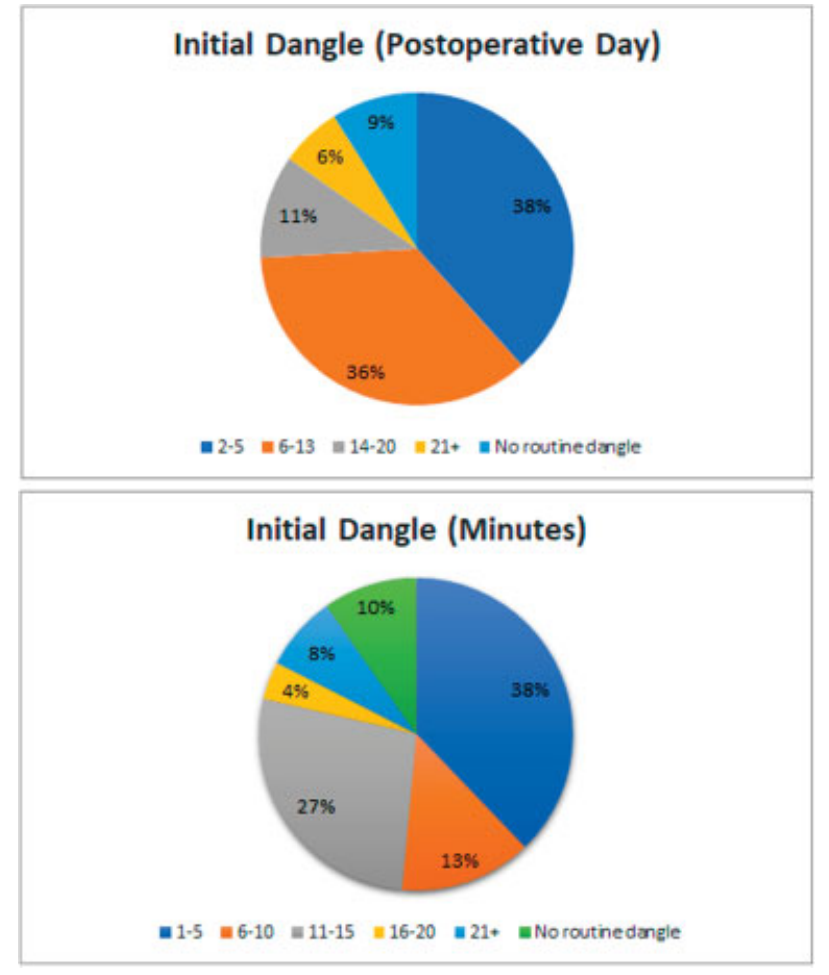

Fig. 2 Outcomes of three surveys regarding dangle protocols $(n=$ number of surgeons). Top: Initial dangle postoperative day $(n=112)$. Bottom: Initial dangle minutes $(n=103)$. the earliest initiation of dangle using traditional anastomotic techniques reviewed. ${ }^{19}$ This group utilized fasciocutaneous perforator flaps and recommended this protocol only in the absence of patient comorbidities such as vascular disease that would place patients at increased risk of complications. ${ }^{19}$ Compressive wrapping was used as an adjunct to aggressive dangle protocols in half of the studies. ${ }^{3,11,12,15,16,18}$ Only one study reported a single incidence of complete flap loss resulting from venous thrombosis that was unable to be salvaged. This occurred prior to initiation of dangle. ${ }^{19}$ Unique aspects examined in these studies are presented here.

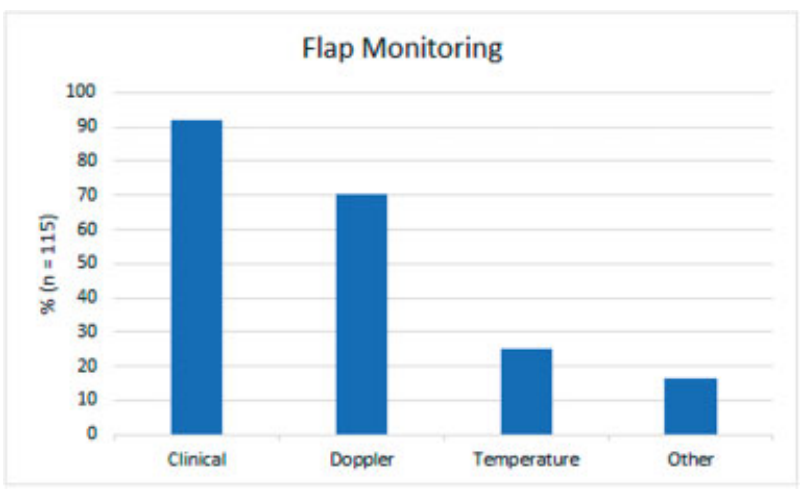

Fig. 3 Outcomes of three surveys regarding methods utilized for flap monitoring $(n=115)$. Note that some use multiple methodologies simultaneously. 
4 Lower-Extremity Free Flap Postoperative Care Soteropulos et al.

\begin{tabular}{|c|c|c|c|c|c|c|c|c|c|c|}
\hline 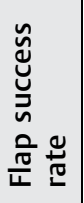 & ळे & ○ें & ○ें & @̊ & ○े & ○े & @̊ & 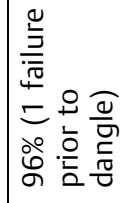 & ஓे & ठํㅇ \\
\hline 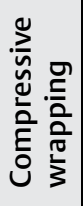 & $\begin{array}{l}\hat{0} \\
0 \\
\text { m } \\
\hat{0} \\
0\end{array}$ & 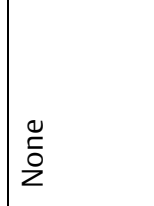 & $\begin{array}{l}5 \\
3 \\
0 \\
2 \\
0 \\
0 \\
0 \\
0\end{array}$ & 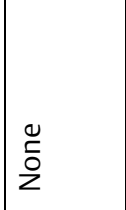 & $\mid \begin{array}{l}0 \\
\tilde{z} \\
z\end{array}$ & $\begin{array}{l}\text { O̊ } \\
\text { Oे }\end{array}$ & $\mid \begin{array}{l}0 \\
\tilde{0} \\
z\end{array}$ & \begin{tabular}{|l}
0 \\
$\check{0}$ \\
$z$
\end{tabular} & 官 & $\frac{\nabla}{\dot{0}}$ \\
\hline 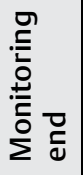 & 응 & $\begin{array}{l}0 \\
\text { İ } \\
z\end{array}$ & 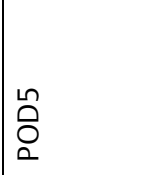 & 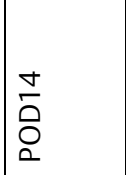 & $\overline{\overline{0}}$ & $\underset{\nabla}{\nabla}$ & 文 & $\begin{array}{l}\text { O̊ } \\
\text { Оे }\end{array}$ & \begin{tabular}{|l}
$\frac{0}{2}$ \\
$\frac{0}{\cdot \frac{\pi}{5}}$ \\
$>$
\end{tabular} & \begin{tabular}{l}
$\circ$ \\
$\Xi$ \\
$\circ$ \\
\hdashline
\end{tabular} \\
\hline 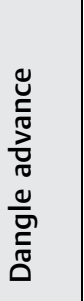 & 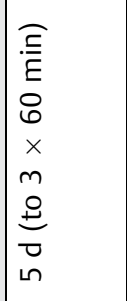 & $\begin{array}{l}0 \\
\check{\tilde{z}} \\
z\end{array}$ & 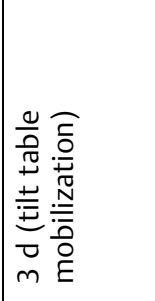 & 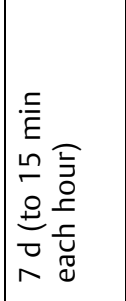 & 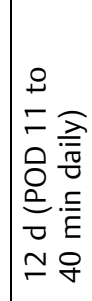 & 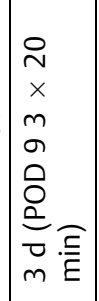 & 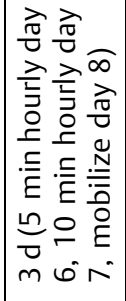 & 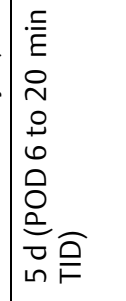 & \begin{tabular}{|l}
$\frac{0}{0}$ \\
$\frac{0}{\cdot \frac{\pi}{5}}$ \\
$>$
\end{tabular} & $\mid \begin{array}{l}0 \\
\tilde{0} \\
z\end{array}$ \\
\hline 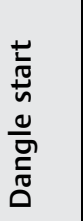 & 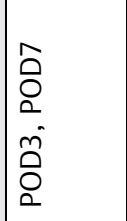 & 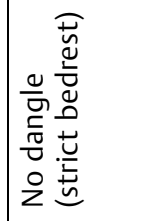 & ỗ & 合 & Oิ̊ & ○ั & $\begin{array}{l}\text { O̊ } \\
\text { О }\end{array}$ & Оิ & $\begin{array}{l}9 \\
\frac{1}{0} \\
0 \\
0\end{array}$ & 官 \\
\hline 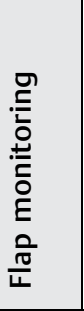 & 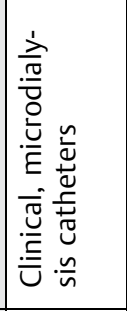 & 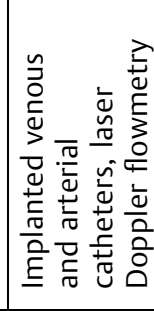 & 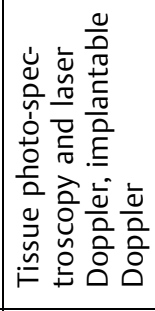 & 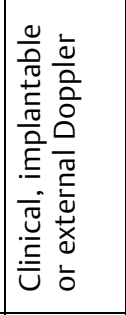 & 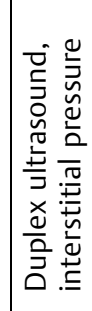 & 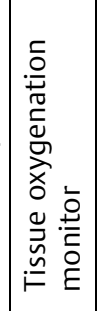 & 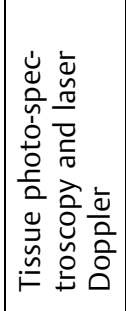 & 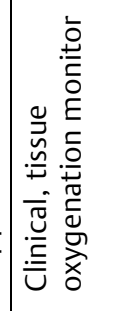 & 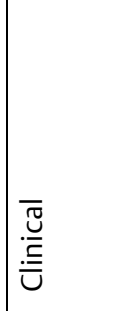 & 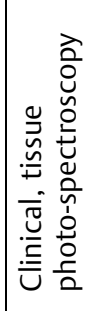 \\
\hline 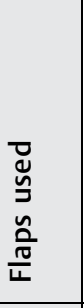 & 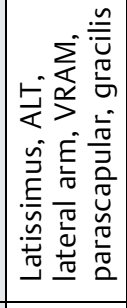 & 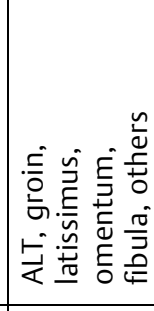 & 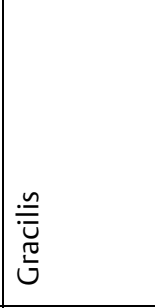 & 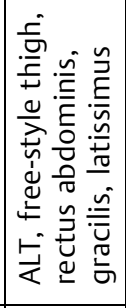 & 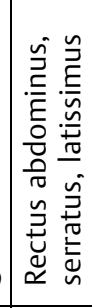 & 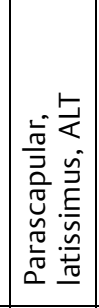 & 高 & 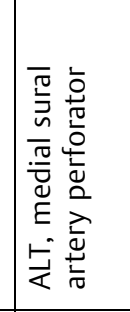 & 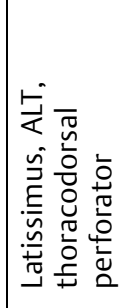 & 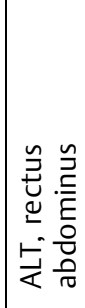 \\
\hline 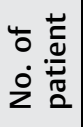 & $\bar{m}$ & 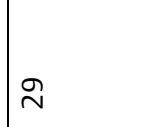 & $\stackrel{\operatorname{Ln}}{\longrightarrow}$ & $\stackrel{\infty}{\sim}$ & $\stackrel{\sim}{N}$ & 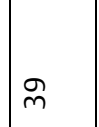 & $\infty$ & $\stackrel{\sim}{N}$ & $\stackrel{m}{r}$ & $\sim$ \\
\hline 量号 & $\underset{\propto}{\check{L}}$ & $\begin{array}{l}\frac{1}{2} \\
0 \\
\frac{1}{0} \\
0\end{array}$ & $\begin{array}{l}\frac{1}{0} \\
\frac{0}{0} \\
\frac{\pi}{0}\end{array}$ & 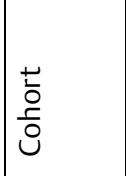 & 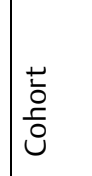 & 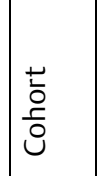 & 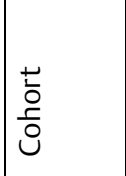 & 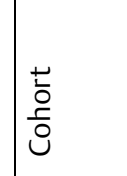 & 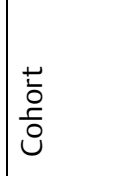 & $\begin{array}{l}\tilde{\tilde{n}} \\
\tilde{u} \\
\tilde{u} \\
\tilde{u}\end{array}$ \\
\hline 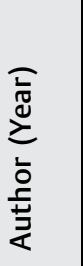 & 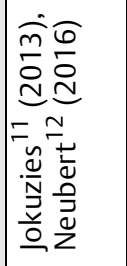 & 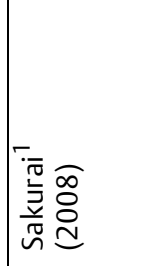 & 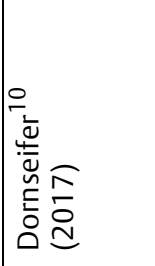 & 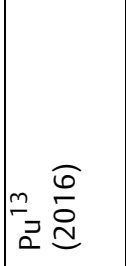 & 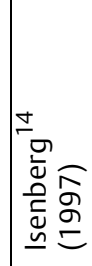 & 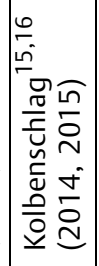 & 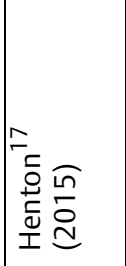 & 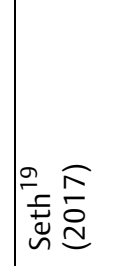 & 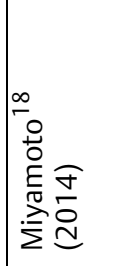 & 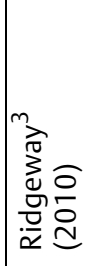 \\
\hline
\end{tabular}




\section{Lower Extremity Flap Physiology without Dangle}

When comparing lower extremity free flaps to those elsewhere on the body, Sakurai et al showed significantly higher venous pressure and lower arterial pressure even when the leg was not in a dependent position. ${ }^{1}$ Even without a dangle protocol in place, the venous pressure was shown to decline from postoperative day three through five. As the arterial venous gradient decreased postoperatively, the perfusion pressure approached that of tissue transferred to other parts of the body. ${ }^{1}$

\section{Influence of Comorbidities on Dangle}

Kolbenschlag et al examined aggressive wrapping and dependency from postoperative day six through nine by monitoring tissue oxygenation. ${ }^{15}$ Tissue oxygenation and hemoglobin concentration showed an initial increase at the beginning of dangle, followed by rapid decrease. During the course of the three-day dangle protocol, tissue oxygenation increased, while the time required to return to pre-dangle values decreased. The effect of comorbidities impacting the vasculature was also examined by Kolbenschlag and colleagues. $^{16}$ Smoking, hypertension, and diabetes were not found to affect flap survival in this study but did affect tissue oxygenation and hemoglobin concentration with dangle. $^{16}$ Smokers showed increased desaturation with dangle, and prolonged recovery period after re-elevation. These differences resolved over the course of the dangle protocol. Diabetic flaps did not demonstrate the same physiologic normalization in the same way as non-diabetics during the course of the dangle protocol, suggesting a need for a longer protocol in these patients. They concluded that initiation of a dangle as early as postoperative day three is safe, but that smoking, diabetes, and hypertension negatively influence flap perfusion, and these patients should be closely monitored. ${ }^{15,16}$

\section{Flow-Through Anastomoses}

To address venous congestion and potentially eliminate the necessity of a dangle protocol, Miyamoto et al have studied the use of flow-through anastomoses for lower extremity free tissue transfer in oncologic reconstruction., ${ }^{4,18}$ They hypothesized that the hemodynamic advantage afforded by flow-through anastomoses of both the artery and vein can effectively mitigate this issue and allow for early dependency and ambulation. In their initial cohort study, 13 patients underwent free flap reconstruction with arterial and venous flow-through anastomoses immediately following soft tissue tumor resection. All patients were mobilized to a wheelchair on postoperative day one. Within the first postoperative week, 12 patients were able to safely dangle, and 10 patients were able to ambulate without flap compromise. ${ }^{18}$ The second study compared traditional end-to-end (ETE) and end-to-side (ETS) anastomoses with flow-through anastomoses. Those patients who underwent flow-through anastomoses of the vein had a $0 \%$ complication rate, while the ETE and ETS had a combined $12.5 \%$ complication rate, although statistical significance was not reached. ${ }^{4}$

\section{Discussion}

\section{Lower Extremity Flap Physiology with Dangle}

Lower extremity flap physiology is unique and requires postoperative management that differs from flaps transferred to other locations. In a healthy lower extremity, the changes in perfusion during dependency are minimal. This is due to vascular compensation resulting from neurologic tone, muscular pumps, collateral flow, and a locally mediated venoarteriolar response (VAR), which serves to limit arterial inflow when venous congestion is high. ${ }^{20}$ The VAR is hypothesized to result from stretch receptors in small veins, which recognize the increase in pressure, and signal for proximal changes in arteriolar tone to reduce blood flow. ${ }^{20}$ This response causes a reduction of blood flow that occurs when venous pressure in a limb rises to higher than $25 \mathrm{~mm} \mathrm{Hg}$. $^{20}$

In a free flap, all compensatory mechanisms are lost except for the locally mediated VAR.,20,21 Although the elevated flap loses the sympathetic nerves, which play a significant role in control of tissue perfusion, the VAR remains intact. ${ }^{11,22}$ The VAR can decrease blood flow to the flap by up to $40 \%$, but this is not enough to prevent the tissue desaturation that occurs due to loss of the other compensatory mechanisms. ${ }^{3}$ The flap desaturates when dependent and takes time to recover to baseline when reelevated, whereas a healthy lower extremity has no difficulty maintaining oxygenation when dependent. ${ }^{15}$

\section{Effect of Dangle Protocols on Flap Physiology}

When dependent, lower extremity free flaps were shown to demonstrate an initial increase in oxygenation and hemoglobin concentration followed by a rapid decrease with prolonged dangle, leading to a reduction in blood flow to the flap. $3,10,15-17$ This corresponds with a decrease in arterial velocity and crosssectional area, an increase in venous cross-sectional area, and a subsequent increase in interstitial pressure. ${ }^{14}$ With a dangle protocol in place, studies show improved saturation and faster recovery of the flap to baseline over time. ${ }^{3,15}$ Instead of subjecting the flap to the challenges of dependency immediately postoperatively, the flap is given time to adapt and ischemia induces neovascularization more quickly. ${ }^{3}$

The majority of aggressive protocols reviewed placed patients on bedrest for 48 hours postoperatively prior to dangle initiation. $3,10-12,14-17$ Physiologically, prior to postoperative day three, the fibrin coverage of the intima at the anastomosis has not fully matured, and the risk of arterial and venous thrombosis is increased. ${ }^{10}$ By day five, a "pseudointima" has formed over the anastomosis. ${ }^{23}$ Surveys showed that surgeon's initiation of dependency varied from three days to four weeks postoperatively (-Figs. 2 and 3). ${ }^{6,8,9}$ Initiation of dangle on postoperative day two was safely performed in one study of fasciocutaneous flaps with traditional anastomotic techniques, suggesting that perhaps this may be safe in a carefully selected patient population. ${ }^{19}$

In terms of flap monitoring, the majority of surgeons agree that clinical examination of the flap is necessary and reliable. When flap intolerance to an initiated protocol is identified, surgeons agree on protocol flexibility. Ambulation 
with crutches or weight bearing as tolerated depending on injury began safely as early as postoperative day 6 after completion of dangle protocols, although many surgeons following long protocols were reluctant to ambulate for many weeks.

\section{Compressive Wrapping}

Compressive wrapping of the flap serves two purposes: (1) reduction of interstitial edema associated with venous congestion of a newly transferred free flap, and (2) induction of ischemic conditioning. $3,11,15,16,24$ Many aggressive dangle protocols employ wrapping with good outcomes. Both dangling and wrapping cause transient hypoxia, which drives vascular endothelial growth factor (VEGF) production, inducing neovascularization, which can increase the rate of flap healing. $3,15,16,24$

\section{Flow-Through Anastomoses}

Using traditional anastomotic techniques, circulation to the flap is affected by limb position. As a result of the VAR, venous congestion results in decreased arterial inflow to prevent tissue congestion; however, this stasis increases the risk of arterial thrombosis and flap failure. With flowthrough anastomoses, the arterial flow can be diverted distally through the open circuit. ${ }^{4,18}$ Venous flow-through anastomoses also increase the flow rate through the anastomoses and preserve the distal pump that can prevent venostasis and increase venous return. These interventions may reduce the risk of flap thrombosis and allow for early mobilization.

\section{Limitations and Future Recommendations}

The majority of studies reviewed were retrospective in nature and had small patient numbers. With a paucity of randomized controlled trials, the influence of patient and flap characteristics on dangle protocols is unable to be accurately evaluated. Many of the studies did not comment on whether the dangle protocol continued beyond the time discussed in the manuscript or following discharge from the hospital. The reviewed studies were largely limited to the traumatic lower extremity reconstruction patient; therefore, results may not be generalizable to other populations. The studies on flow-through anastomoses were limited to oncologic reconstruction, which may not be applicable to the lower extremity trauma patient., ${ }^{48}$ All but a single study reported a $100 \%$ flap success rate, suggesting study numbers were too small to reliably capture failure. ${ }^{19}$ Flaps that are threatened or for which the surgeon has concerns are unlikely to enter a typical dangle protocol. It would be useful to know how many flaps do not enter the typical protocol or fall out of the protocol due to signs of congestion during dangle. Unfortunately, this was often not reported and therefore is a limitation of this review.

A large, multicenter prospective randomized controlled trial would be necessary to truly understand the implications of a dangle protocol on flap survival, hospital stay, return to ambulation, and cost of care. A study of this magnitude could also evaluate the effect of patient comorbidities and flap types on these outcomes. Furthermore, this may better capture how free flaps for lower extremity trauma are affected by variables such as traumatic mechanism, zone of injury, concomitant injury, and comorbidities. Theoretically, more aggressive protocols would reduce hospital stay and cost associated with lower extremity free tissue transfer. However, higher level of evidence data would be helpful to risk stratify patients into those who would be able to tolerate aggressive dangle protocols versus those who may require more time for physiologic equilibration.

\section{Conclusions}

Physiology of lower extremity free flaps is unique from flaps in other locations. This is due to the loss of complex physiologic mechanisms that regulate blood flow in the normal lower limb. Various dangle protocols have been developed to reduce the risk of flap failure, and compressive wrapping is a useful adjunct. Based on this systematic review, early initiation of a dangle protocol does not appear to negatively impact flap survival. Many surgeons agree that clinical monitoring is sufficient; there is no consensus on the utility of adjunct monitoring techniques. Weight bearing with close flap monitoring may begin after completion of dangle protocol, if not prevented by restrictions due to orthopedic injury. Further research, including large volume, randomized controlled trials, is needed to develop better evidence-based guidelines to maximize successful free flap outcomes while minimizing the excess time, cost, and morbidity associated with prolonged hospitalization and bedrest.

\section{Conflict of Interest}

None.

\section{References}

1 Sakurai H, Yamaki T, Takeuchi M, Soejima K, Kono T, Nozaki M. Hemodynamic alterations in the transferred tissue to lower extremities. Microsurgery 2009;29(02):101-106

2 Fischer JP, Wink JD, Nelson JA, et al. A retrospective review of outcomes and flap selection in free tissue transfers for complex lower extremity reconstruction. J Reconstr Microsurg 2013;29 (06):407-416

3 Ridgway EB, Kutz RH, Cooper JS, Guo L. New insight into an old paradigm: wrapping and dangling with lower-extremity free flaps. J Reconstr Microsurg 2010;26(08):559-566

4 Fujiki M, Miyamoto S, Sakuraba M. Flow-through anastomosis for both the artery and vein in leg free flap transfer. Microsurgery 2015;35(07):536-540

5 Chow SP, Chen DZ, Gu YD. The significance of venous drainage in free flap transfer. Plast Reconstr Surg 1993;91(04):713-715

6 Rohde C, Howell BW, Buncke GM, et al. A recommended protocol for the immediate postoperative care of lower extremity free-flap reconstructions. J Reconstr Microsurg 2009;25(01):15-19

7 Moher D, Liberati A, Tetzlaff J, Altman DG; PRISMA Group. Preferred reporting items for systematic reviews and meta-analyses: the PRISMA statement. PLoS Med 2009;6(07):e1000097

8 Xipoleas G, Levine E, Silver L, Koch RM, Taub PJ. A survey of microvascular protocols for lower extremity free tissue transfer II: postoperative care. Ann Plast Surg 2008;61(03):280-284 
9 O'Neill JK, Greenwood AJ, Khan U. A survey of U.K. units and a suggested protocol for free flap reconstruction of the lower limb: follow-up and management in the first postoperative week. J Reconstr Microsurg 2010;26(09):601-606

10 Dornseifer U, Kleeberger C, Kargl L, et al. Perfusion controlled mobilization after lower extremity free flaps-pushing the limits of time and intensity. J Reconstr Microsurg 2017;33(03):179-185

11 Jokuszies A, Neubert N, Herold C, Vogt PM. Early start of the dangling procedure in lower extremity free flap reconstruction does not affect the clinical outcome.J Reconstr Microsurg 2013;29 (01):27-32

12 Neubert N, Vogt PM, May M, et al. Does an early and aggressive combined wrapping and dangling procedure affect the clinical outcome of lower extremity free flaps? A randomized controlled prospective study using microdialysis monitoring. J Reconstr Microsurg 2016;32(04):262-270

$13 \mathrm{Pu}$ LL. A comprehensive approach to lower extremity free-tissue transfer. Plast Reconstr Surg Glob Open 2017;5(02):e1228

14 Isenberg JS, Siegal A, Sherman R. Quantitative evaluation of the effects of gravity and dependency on microvascular tissue transfer to the lower limb, with clinical applications. J Reconstr Microsurg 1997;13(01):25-29

15 Kolbenschlag J, Bredenbroeker P, Daigeler A, et al. Changes of oxygenation and hemoglobin-concentration in lower extremity free flaps during dangling. J Reconstr Microsurg 2014;30(05):319-328

16 Kolbenschlag J, Bredenbroeker P, Lehnhardt M, et al. Advanced microcirculatory parameters of lower extremity free flaps during dangling and their influencing factors. J Reconstr Microsurg 2015; 31(07):500-507

17 Henton JM, Simmons JM, Hettiaratchy S, Jain A. Perfusion dynamics in lower limb reconstruction: investigating postoperative recovery and training using combined white light photospectroscopy and laser Doppler $\left(\mathrm{O} 2 \mathrm{C}\left({ }^{\circledR}\right)\right)$. J Plast Reconstr Aesthet Surg 2015;68(09):1286-1292

18 Miyamoto S, Kayano S, Fujiki M, Chuman H, Kawai A, Sakuraba M. Early mobilization after free-flap transfer to the lower extremities: preferential use of flow-through anastomosis. Plast Reconstr Surg Glob Open 2014;2(03):e127

19 Seth AK, Diamond S, Iorio ML. Outcomes of an early protocol for dependent conditioning in lower extremity microsurgical free flaps. J Reconstr Microsurg 2017;33(09):670-678

20 Okazaki K, Fu Q, Martini ER, et al. Vasoconstriction during venous congestion: effects of venoarteriolar response, myogenic reflexes, and hemodynamics of changing perfusion pressure. Am J Physiol Regul Integr Comp Physiol 2005;289(05):R1354-R1359

21 Hassan AA, Tooke JE. Mechanism of the postural vasoconstrictor response in the human foot. Clin Sci (Lond) 1988;75(04):379-387

22 Henriksen O. Local sympathetic reflex mechanism in regulation of blood flow in human subcutaneous adipose tissue. Acta Physiol Scand Suppl 1977;450:1-48

23 Harashina T, Fujino T, Watanabe S. The intimal healing of microvascular anastomoses. Plast Reconstr Surg 1976;58(05):608-613

$24 \mathrm{Kim}$ YW, Byzova TV. Oxidative stress in angiogenesis and vascular disease. Blood 2014;123(05):625-631 